\title{
Epidural Fat-Derived Mesenchymal Stem Cell: First Report of Epidural Fat-Derived Mesenchymal Stem Cell
}

\author{
Gun Woo Lee ${ }^{1}$, Min-Soo Seo ${ }^{2}$, Kyung-Ku Kang ${ }^{2}$, Se-Kyung $\mathrm{Oh}^{2}$ \\ ${ }^{1}$ Department of Orthopaedic Surgery, Spine Center, Yeungnam University Medical Center, Yeungnam University College of Medicine, Daegu, Korea \\ ${ }^{2}$ Labaratory Animal Center, Daegu-Gyeongbuk Medical Innovation Foundation, Daegu, Korea
}

Study Design: Experimental study.

Purpose: To determine whether epidural fat (EF) tissue contains mesenchymal stem cells (MSC).

Overview of Literature: Spine surgeons are unaware of the contents of EF tissue and the reason for its presence between the ligamentum flavum and the dura mater; therefore, EF tissues are routinely eliminated during surgical procedures. However, EF removal causes certain postoperative problems, such as post-laminectomy syndrome. We hypothesized that the EF tissue may play a significant supportive role for the neural structures and other nearby conditions.

Methods: EF tissues were obtained from consenting patients $(n=3)$ during posterior decompression surgery of the lumbar spine. The primary cells were isolated and cultured as per previously described methods with some modifications, and the cell morphology and cumulation were examined. Thereafter, reverse transcription-polymerase chain reaction (RT-PCR), a fluorescence-activated cell sorting (FACS) analysis, and differentiation potency for differentiation into osteoblasts, chondroblasts, and adipocytes were investigated to identify whether the cells derived from EF are MSC.

Results: The cells from the EF tissue had a fibroblast or neuron-like morphology that persisted until the senescence at p18. MSCspecific genes, such as OCT4, SOX2, KLF4, MYC, and GAPDH were expressed in the RT-PCR study, while MSC-specific surface markers such as CD105, CD90, and CD73 were exhibited in the FACS analysis. The differentiation properties of EF-MSC for differentiation into the three types of cells (osteoblast, chondroblast, and adipocyte) were also confirmed.

Conclusions: Based on the cell culture, FACS analysis, RT-PCR analysis, and differentiation potent outcomes, all the features of the cells corresponded to MSC. This is the first study to identify EF-MSC derived from the EF tissue.

Keywords: Epidural fat; Mesenchymal stem cells; Reverse transcription-polymerase chain reaction; Fluorescence-activated cell sorting; Differentiation

\section{Introduction}

During posterior spine surgery, spine surgeons always encounter yellowish epidural fat (EF) that covers the dura mater and the nerve root after ligamentum flavum (LF) resection. However, the role and function of EF remains

Received Aug 22, 2018; Revised Oct 4, 2018; Accepted Nov 3, 2018

Corresponding author: Gun Woo Lee

Department of Orthopaedic Surgery, Spine Center, Yeungnam University Hospital, Yeungnam University College of Medicine, 170 Hyeonchung-ro, Nam-gu, Daegu 42415, Korea

Tel: +82-53-620-3642, Fax: +82-53-628-4020, E-mail: gwlee1871@gmail.com 
unclear. Spine surgeons have limited knowledge regarding EF; therefore, no effort is made to preserve it during surgical procedures, resulting in its loss and exposure of the dura mater and the nerve roots [1].

What is the role of EF, and why is it located between the LF and the dura mater (spinal cord) or the nerve root? Few anatomical and histologic studies have concluded the following: (1) EF is also an adipose tissue; however, its histologic morphology and shape differ from those of subcutaneous fat [2-5]; and (2) the EF may enable smooth sliding of the nervous structures, such as the dura mater and the nerve roots from the LF and other stable structures $[1,5,6]$. However, to our knowledge, no studies have investigated EF in detail, especially focusing on the mesenchymal stem cells (MSC) in the EF tissue.

The authors hypothesized that the EF may have critical functions for the spinal cord, nerves, dura mater, and surrounding structures. In addition, if EF is in fact an adipose tissue, MSC with specific regenerative and antiinflammatory functions for the surrounding structures may be present [7-10]. According to this hypothesis, we have conducted several experimental studies on EF. The current study, the first and fundamental report of these experimental and clinical studies, focuses on 'whether EF tissue contains MSC.'

\section{Materials and Methods}

\section{Epidural fat-derived stem cell isolation and culture}

This study was approved by the Institutional Review Board and Ethical Committee of the Yeungnam University Medical Center (YUMC IRB no., 2017-07-032). The EF was obtained from consenting patients $(n=3)$ during posterior decompression surgery of the lumbar spine performed at our center. The specimens were placed in sterile tubes, stored at $4^{\circ} \mathrm{C}$, and brought to the Laboratory Animal Center of the Daegu-Gyeongbuk Medical Innovation Foundation to be processed within 12 hours.

The primary cells were isolated and cultured as previously described with some modifications [11]. Briefly, the collected fat tissues were washed with phosphate-buffered saline (PBS; Gibco Invitrogen, Carlsbad CA, USA) 3-4 times to remove blood and debris. The washed tissues were minced using scissors and surgical blades. The tissues were incubated with collagenase type I $(2 \mathrm{mg} / \mathrm{mL}$; Sigma-Aldrich, St. Louis, MO, USA) for 1-2 hours. The digested samples were sieved with a cell strainer (pore size $=70 \mu \mathrm{m}$, Falcon; BD Biosciences, Coning, NY, USA) and then washed with PBS and centrifuged at $350 \times \mathrm{g}$ for 10 minutes for cell isolation. The cell pellet was suspended in low glucose Dulbecco's modified Eagle's medium (Invitrogen, Carlsbad, CA, USA) containing 10\% fetal bovine serum (Invitrogen). The cells were cultured in a $5 \% \mathrm{CO}_{2}$ incubator with culture medium changes thrice a week.

\section{Cumulative population doubling level analysis}

The proliferation ability of the cultured cells was measured, as previously described, with some modifications $[12,13]$. The growth and proliferation ability of the cultured cells were estimated via the cumulative population doubling level (CPDL) using the formula $\mathrm{CPDL}=\ln (\mathrm{Nf} /$ $\mathrm{Ni}) \ln 2$, where $\mathrm{Ni}$ is the initial number of cells seeded, $\mathrm{Nf}$ is the final number of harvested cells, and $\ln$ is the natural $\log$. The cells $\left(3 \times 10^{5}\right)$ were seeded in triplicate using 6-well culture dishes and subcultured for 3 days. Thereafter, the cells were counted and reseeded. To estimate the CPDL, the population doubling of each passage was measured and added to the previous cell population double levels.

\section{Reverse transcription-polymerase chain reaction}

Total RNA was extracted from the cultured cells using an RNeasy mini kit and RNase-Free DNase set (QIAGEN, Hilden, Germany) as per the manufacturer's protocol. RNA concentrations were measured by the ratio of absorbance at $260 \mathrm{~nm}$ and $280 \mathrm{~nm}$ using a spectrophotometer. Complementary DNA (cDNA) was prepared using $1 \mathrm{mg}$ of total RNA for reverse transcription (RT) using SuperScript III reverse transcriptase (Invitrogen, Groningen, Netherlands). The cDNA was amplified with polymerase chain reaction (PCR) with Takara Ex Taq (Takara Bio, Kusatsu, Japan). The PCR primers are shown in Table 1. The PCR products were separated on a $1.5 \%$ agarose gel and visualized with ethidium bromide. Quantitative RT-PCR was performed by mixing the cDNA with primers and SYBR Green I Master Mix (Roche Diagnostics, Mannheim, Germany). Quantitative RT-PCR was performed using a LightCycler 480 system with supplied software (Roche Diagnostics), as per the manufacturer's instructions. The RNA expression levels were compared after normalization to endogenous glyceraldehyde-3phosphate dehydrogenase (GAPDH). 
Table 1. Primer sequences

\begin{tabular}{lll} 
Genes & Forward primer $\left(5^{\prime}-3^{\prime}\right)$ & \multicolumn{1}{c}{ Reverse primer $\left(5^{\prime}-3^{\prime}\right)$} \\
\hline OCT4 & CTTCAGGAGATATGCAAAGCA & ACACTCGGACCACATCCTTC \\
SOX2 & TTGCCAATATTTTTCAAGGAGA & CAAGACCACAGAGATGGTTCG \\
\hline KLF4 & ATTAATGAGGCAGCCACCTG & GGAAGCACTGGGGGAAGTC \\
MYC & GCGACTCTGAGGAGGACAA & TGCGTAGTTGTGCTGATGTG \\
\hline GAPDH & CGCTGAGTACGTCGTGGAGT & GGAGGCATTGCTGATGATCT \\
\hline
\end{tabular}

\section{Fluorescence-activated cell sorting analysis}

To determine the expression of cell surface markers, the cultured cells were stained and analyzed using flow cytometry. Cell staining was performed as per the manufacturer's cell surface immunofluorescence staining protocol (Biolegend, San Diego, CA, USA). Briefly, the cells were washed twice with PBS and detached using 0.25\% trypsin/ ethylenediaminetetraacetic acid. The detached cells were collected, washed with PBS, and divided into groups for antibody staining. Each group contained about $1 \times 10^{5}$ cells for staining. The antibodies used to detect the cell surface markers included CD105, CD90, CD73, CD45, CD34, and CD14 (BioLegend; all antibodies for anti-human). All the antibodies were conjugated with fluorescein dye. The staining was conducted for 30 minutes at $4^{\circ} \mathrm{C}$ with antibody incubation. After the incubation, the cells were washed twice with PBS. Fluorescence-activated cell sorting (FACS) analysis was performed with a FACS Calibur cytometer (BD Biosciences, San Jose, CA, USA).

\section{Osteogenesis}

To confirm the osteogenic differentiation ability of human EF-MSC, the cells were treated with an osteogenic differentiation medium (StemPro Osteogenesis Differentiation kit, Gibco Invitrogen). For osteogenic differentiation, the cells were cultured in 6-well culture plates. At 70\%-80\% cellular confluence, the culture medium was changed to an osteogenic differentiation medium. The differentiation medium was changed twice weekly for 3 weeks. The differentiated cells were stained with von Kossa and alizarin red S staining to confirm calcium deposition. For von Kossa staining, the cells were stained with 5\% silver nitrate for 1 hour and exposed to ultraviolet light. Following incubation, the differentiated cells were treated with 5\% sodium thiosulfate for 3 minutes and counterstained with nuclear red stain for 3 minutes. For alizarin red S staining, the differentiated cells were fixed with $70 \%$ ethanol for 1 hour at $4^{\circ} \mathrm{C}$. After fixation, the cells were washed with distilled water and stained with alizarin red S (Sigma-Aldrich) for 10 minutes at room temperature. Thereafter, the cells were washed 3-4 times with distilled water. Alizarin red Sstained cells were solubilized in cetylpyridinium chloride (Sigma-Aldrich) for 1 hour. A spectrophotometer was used to measure the absorbance of the solubilized cells at $570 \mathrm{~nm}$.

\section{Adipogenesis}

To confirm the adipogenic differentiation ability of human EF-MSC, the cells were treated with adipogenic differentiation medium (StemPro Adipogenesis Differentiation kit, Gibco Invitrogen). When the cells reached 70\%-80\% confluence, the adipogenic differentiation medium was used for 3 weeks. After adipogenic differentiation, oil red O staining was performed to detect the lipid droplets. The cells were fixed with $10 \%$ formalin for 1 hour and washed with $60 \%$ isopropanol. After fixation, the cells were stained with oil red $\mathrm{O}$ for 10 minutes. The oil red O-stained cells were solubilized in 100\% isopropanol, and the absorbance of the solubilized solution was measured at $500 \mathrm{~nm}$ using a spectrophotometer.

\section{Chondrogenesis}

To detect the chondrogenesis differentiation ability of human EF-MSC, the cells were treated with chondrogenic differentiation medium (StemPro Chondrogenesis Differentiation kit, Gibco Invitrogen). The cells $\left(2 \times 10^{5}\right)$ were seeded in a $15-\mathrm{mL}$ polypropylene tube and spun down in a centrifuge. The cells were treated with the chondrogenic differentiation medium $(1 \mathrm{~mL})$ for 3 weeks. The differentiation medium was changed 3 times every week. 
After differentiation, the cells were confirmed the pellet formation. The pellet was embedded in a paraffin block, sectioned, and stained with toluidine blue to confirm the chondrogenic differentiation ability.

\section{Results}

\section{Stem cell properties of epidural fat-derived mesen- chymal stem cells}

After 3 weeks of culturing of MSC isolated from human $\mathrm{EF}$ tissues (EF-MSC), plastic-adherent cells with a unique morphology were obtained. The EF-MSC exhibited neuronal or fibroblast-like bipolar morphologies until senescence at p18 (Fig. 1A). Throughout the culturing, they underwent a mean 15.8 doublings and produced $6.9 \times 10^{16}$ cells (Fig. 1B).

In the RT-PCR analysis, EF-MSC at p4 expressed stem cell-specific genes, including OCT4, SOX2, KLF4, MYC, and GAPDH; however, no expression was observed in the control group (Fig. 1C). The FACS analysis showed that EF-MSC at p4 expressed stem cell-specific surface markers of CD105, CD90, and CD73 but not those of CD45, CD34, and CD14 (Fig. 2).

\section{Differentiation potentials of epidural fat-derived mesenchymal stem cells}

EF-MSC cultured in appropriate differentiation media showed osteogenic (with alizarin red S stain and von Kossa stain), adipogenic (with oil red O stain), and chondrogenic (with toluidine blue stain) differentiation potentials (Fig. 3).

\section{Discussion}

Currently, spine surgeons remain unaware regarding what $\mathrm{EF}$ tissue is and why it is located in the space between the LF and the neural structures. However, EF tissues are eliminated completely after surgical procedures, and spine surgeons have suggested that full exposure of the dura mater and nerve root with EF tissue removal indicate full decompression and surgical success. However, several studies have documented some problems after spine surgery, such as post-laminectomy syndrome, caused to the lack of knowledge among surgeons about the role of EF tissue $[1,2,5,6]$. We hypothesized that the EF tissue may also play a significant role in the neural and other nearby structures. Thus, we conducted several experimental and clinical studies on EF tissue. The current fundamental and basic study focused on whether EF tissues contain EFMSC.

To certify whether the cells derived from certain tissues are MSC, several steps need to be completed. The current study revealed that the cells had a fibroblast- or neuronlike morphology typically observed in MSC that remained until senescence at p18. MSC-specific genes such as OCT4, SOX2, KLF4, MYC, and GAPDH were expressed in the RT-PCR analysis, and MSC-specific surface markers, such as CD105, CD90, and CD73 were exhibited in the FACS analysis. Finally, and most importantly, the cells' adipogenic, chondrogenic, and osteogenic differentiation properties were verified (Fig. 3). Based on the outcomes, we concluded that EF tissues possess MSC (EF-MSC), and the EF-MSC may have specific regenerative properties for the nervous or other nearby structures and anti-inflammatory properties for the epidural space or the surround-
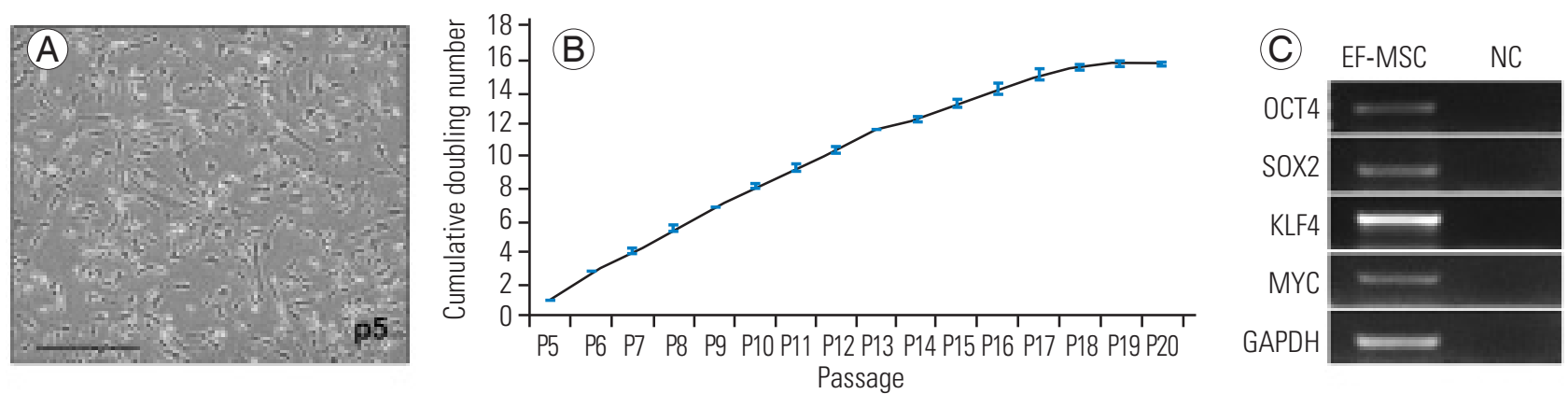

Fig. 1. Stem cell properties of EF-MSC. (A) Morphology of EF-MSC at p5 (scale bar=50 $\mu \mathrm{m}$ ). (B) Cumulative doubling number of EF-MSC throughout ex vivo expansions. (C) Expression of stem cell-specific genes by EF-MSC in the reverse transcription-polymerase chain reaction analysis. p, passage; EF-MSC, epidural fat-mesenchymal stem cell; NC, negative control. 

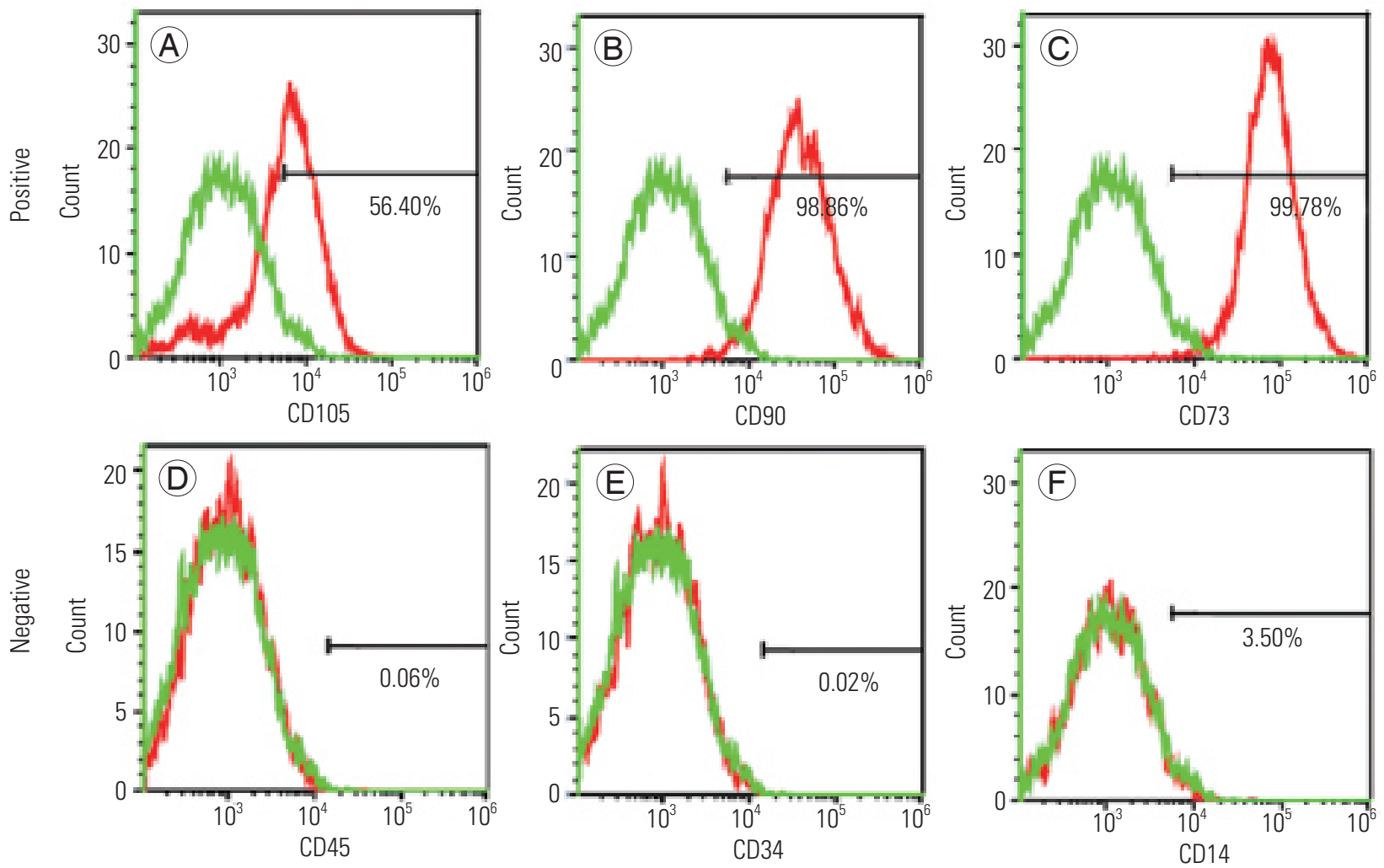

Fig. 2. Flow cytometric analyses of human EF-MSC. EF-MSC at passage 4 were strongly positive for CD105 (A), CD90 (B), and CD73 (C), and negative for CD45 (D), CD34 (E), and CD14 (F) antigens. EF-MSC, epidural fat-derived mesenchymal stem cell.
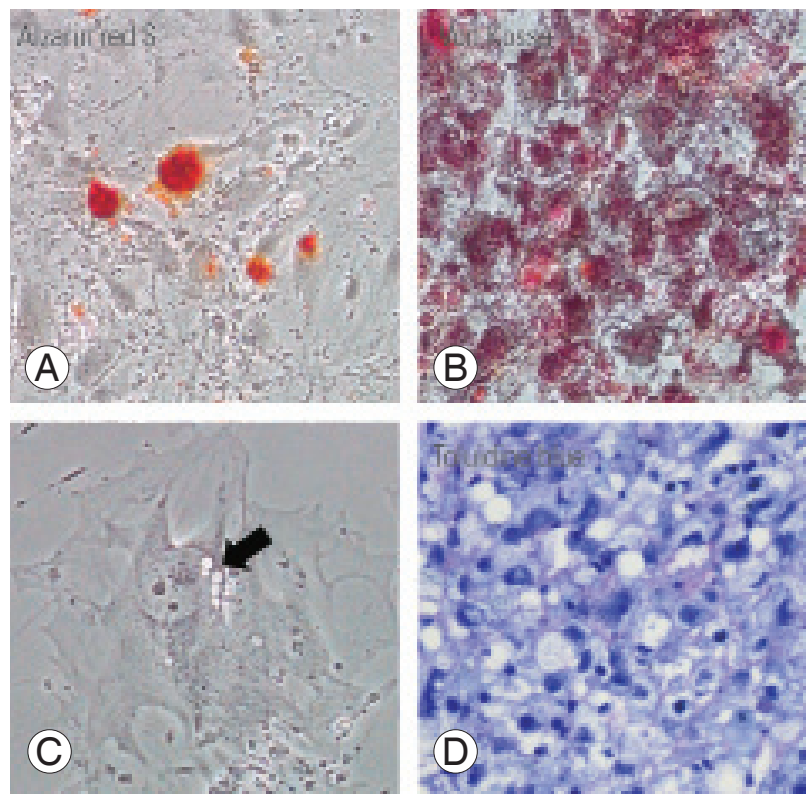

Fig. 3. (A-D) Differentiation potential of epidural fat-derived mesenchymal stem cell at p4 into osteoblasts, adipocytes, and chondroblasts (black arrow: adipocyte). (A) Alizarin red S stain (x100). (B) Von Kossa stain (x100). (C) Adipocyte, unstained (x100). (D) Toluidine blue stain (x100). ing structures.

Thus far, few studies have evaluated the histologic features of EF tissues or its impact on epidural adhesion or scarring $[2,4,5]$; however, no studies have investigated EFderived MSC and their specific functions. The MSC derived from adipose tissues have the following two specific functions; regenerative effect and an anti-inflammatory effect that are more effective for nearby structures [710,14]. Thus, EF-MSC may perform some of the following functions. First, the EF-MSC may impact the regeneration of nearby neural structures, such as the spinal cord. Spinal cord injuries are occasionally induced by trauma or disease; however, no definite treatment method has been established for them. Many experimental studies using MSC have been conducted [15-17]; however, they are mostly induced from subcutaneous fat tissue owing to the ease in harvesting and availability, or other originated stem cells. Second, EF-MSC may also affect peridural adhesion or scarring by MSC-induced anti-inflammatory properties $[10,16,18,19]$. Postsurgical epidural scarring or adhesions are considered significant issues for clinical impairments, such as the post-laminectomy syndrome $[1,6]$ that may be 
caused by the removal of the EF tissue during spine surgery. Considering the anti-inflammatory effects of MSC, the preservation of EF tissue may reduce specific epidural adhesion-related conditions. In fact, the authors have performed several related experimental and clinical studies, and we shall present the findings in the near future.

The current study had certain limitations. First, we described the stem cell features of EF-MSC without comparing it to other MSC, such as subcutaneous tissue-derived MSC. Most MSC studies conducted thus far have used subcutaneous tissue-derived MSC in fat tissues; therefore, the comparison between the EF-MSC and those derived from subcutaneous tissue may be instructive. We are aware of this limitation; therefore, we intend to conduct a comparative study of the two MSC in the future. Second, the unique features of EF-MSC should be investigated experimentally in future studies. Moreover, as described in the 'Discussion' section, the EF tissue may have a critical influence on the patients' clinical outcomes. The current study was fundamental for further experimental and clinical studies regarding EF-MSC; therefore, additional studies are important for defining its significance. Despite these limitations, the study has significant strengths. This was the first study to identify EF-MSC that may be an important stem cell source for specific pathologies and may have a critical influence on spine conditions. Thus, we believe that this study can be a cornerstone for further studies on EF-MSC.

\section{Conclusions}

To our knowledge, this is the first study to investigate EF-MSC. Based on the cell culture, FACS analysis, RTPCR analysis, and differentiation potential (adipogenesis, chondrogenesis, and osteogenesis) outcomes of this study, all the features of the cells corresponded to MSC, and we defined EF-MSC in the EF tissue. Further experimental and clinical studies with EF-MSC are warranted to identify the significance of our findings.

\section{Conflict of Interest}

No potential conflict of interest relevant to this article was reported.

\section{Acknowledgments}

This work was supported by the National Research Foundation of Korea grant funded by the Korean Government and Ministry of Education (2017R1D1A1B03030530). The funder had no role in the study design, data collection and analysis, and study progression.

\section{References}

1. Xu J, Chen Y, Yue Y, Sun J, Cui L. Reconstruction of epidural fat with engineered adipose tissue from adipose derived stem cells and PLGA in the rabbit dorsal laminectomy model. Biomaterials 2012;33:696573.

2. Beaujeux R, Wolfram-Gabel R, Kehrli P, et al. Posterior lumbar epidural fat as a functional structure?: histologic specificities. Spine 1997;22:1264-8.

3. Reina MA, Pulido P, Castedo J, et al. Epidural fat in various diseases: contribution of magnetic resonance imaging and potential implications for neuro axial anesthesia. Rev Esp Anestesiol Reanim 2007;54:17383.

4. Reina MA, Pulido P, Castedo J, Villanueva MC, Lopez A, Sola RG. Characteristics and distribution of normal human epidural fat. Rev Esp Anestesiol Reanim 2006;53:363-72.

5. Reina MA, Franco CD, Lopez A, De Andres JA, van Zundert A. Clinical implications of epidural fat in the spinal canal: a scanning electron microscopic study. Acta Anaesthesiol Belg 2009;60:7-17.

6. Lin CY, Liu TY, Chen MH, Sun JS, Chen MH. An injectable extracellular matrix for the reconstruction of epidural fat and the prevention of epidural fibrosis. Biomed Mater 2016;11:035010.

7. Baer PC. Adipose-derived stem cells and their potential to differentiate into the epithelial lineage. Stem Cells Dev 2011;20:1805-16.

8. Huang SJ, Fu RH, Shyu WC, et al. Adipose-derived stem cells: isolation, characterization, and differentiation potential. Cell Transplant 2013;22:701-9.

9. Kang HM, Kim J, Park S, et al. Insulin-secreting cells from human eyelid-derived stem cells alleviate type I diabetes in immunocompetent mice. Stem Cells 2009;27:1999-2008.

10. Paschos NK, Sennett ML. Update on mesenchymal stem cell therapies for cartilage disorders. World J 
Orthop 2017;8:853-60.

11. Bunnell BA, Flaat M, Gagliardi C, Patel B, Ripoll C. Adipose-derived stem cells: isolation, expansion and differentiation. Methods 2008;45:115-20.

12. Cristofalo VJ, Allen RG, Pignolo RJ, Martin BG, Beck JC. Relationship between donor age and the replicative lifespan of human cells in culture: a reevaluation. Proc Natl Acad Sci U S A 1998;95:10614-9.

13. Kim J, Kang HM, Kim H, et al. Ex vivo characteristics of human amniotic membrane-derived stem cells. Cloning Stem Cells 2007;9:581-94.

14. Guilak F, Lott KE, Awad HA, et al. Clonal analysis of the differentiation potential of human adiposederived adult stem cells. J Cell Physiol 2006;206:22937.

15. Nagoshi N, Khazaei M, Ahlfors JE, et al. Human spinal oligodendrogenic neural progenitor cells promote functional recovery after spinal cord injury by axonal remyelination and tissue sparing. Stem Cells Transl Med 2018;7:806-18.

16. Wu GH, Shi HJ, Che MT, et al. Recovery of paralyzed limb motor function in canine with complete spinal cord injury following implantation of MSC-derived neural network tissue. Biomaterials 2018;181:15-34.

17. Glicksman MA. Induced pluripotent stem cells: the most versatile source for stem cell therapy. Clin Ther 2018;40:1060-5.

18. Goodarzi P, Larijani B, Alavi-Moghadam S, et al. Mesenchymal stem cells-derived exosomes for wound regeneration. Adv Exp Med Biol 2018;1119:119-31.

19. Oberringer $M$, Bubel $M$, Jennewein $M$, et al. The role of adipose-derived stem cells in a self-organizing $3 \mathrm{D}$ model with regard to human soft tissue healing. Mol Cell Biochem 2018;445:195-210. 